\title{
IMWG Imaging plus MRD-negative
}

National Cancer Institute

\section{Source}

National Cancer Institute. IMWG Imaging plus MRD-negative. NCI Thesaurus. Code C159803.

Minimal residual disease negativity as defined by next-generation flow cytometry or nextgeneration sequencing plus disappearance of every area of increased tracer uptake found at baseline or a preceding PET/CT or decrease to less mediastinal blood pool standard uptake value or decrease to less than that of surrounding normal tissue. 